\title{
Role of MRI in the diagnosis of adult traumatic and obstetric brachial plexus injury compared to intraoperative findings
}

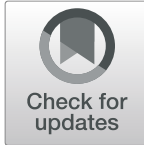

Doria Mohammed Gad ${ }^{1 *}$, Mostafa Thabet Hussein ${ }^{1}$, Nagham Nabil Mahmoud Omar', Mohamed Mostafa Kotb², Mohamed Abdel-Tawab ${ }^{1}$ and Hazem Abu Zeid Yousef ${ }^{1}$

\begin{abstract}
Background: Brachial plexus injury occurs following birth trauma or adult trauma as well, surgical repair is important to regain upper limb function, and preoperative evaluation with MRI is important and considered the accurate and safe imaging modality.

Thirty-seven patients with clinically suspected obstetric (15 patients) or adult traumatic (22 patients) brachial plexus injury were included in our study; all of them underwent MRI examination including T1WI, T2WI, STIR, DWIBS, 3D STIR SPACE, and MR myelography sequences.

Results: In obstetric cases, MRI sensitivity, specificity, and accuracy for preganglionic injury were $63 \%$, 89\%, and 82\%, respectively, while for postganglionic lesions, MRI sensitivity, specificity, and accuracy were 60\%, 99\%, and 95\%, respectively. In adult cases, MRI sensitivity, specificity, and accuracy for preganglionic injury were 96\%, 95\%, and 95\% respectively, while for postganglionic injury, MRI sensitivity, specificity, and accuracy were 60\%, 100\%, and 99\%, respectively.
\end{abstract}

Conclusion: MRI represents a safe, non-invasive, diagnostic modality having the multiplanar capability and better soft tissue characterization.

Keywords: Brachial plexus injury, Adult traumatic, Obstetric, MRI

\section{Background}

Newborns' brachial plexus injury occurs in $0.5-4$ per 1000 vaginal deliveries [1-3]. Approximately $80 \%$ of them recover spontaneously by the age of 1 year old [3]. In babies with a permanent injury, shoulder and elbow contractures usually occur following muscle changes $[4,5]$. Without nerve repair, at least one third of those patients may develop posterior instability, subluxation, and deformity of the glenohumeral joint [1]. Infants with root avulsions have a poorer prognosis compared with infants without [6].

Traumatic brachial plexus injuries affect $1 \%$ of patients involved in major trauma [7]. Brachial plexopathy may cause everlasting disability [8-12], pain [12-14],

\footnotetext{
* Correspondence: doriamohammed61@gmail.com

${ }^{1}$ Department of Radiology, Assiut University, Assiut, Egypt

Full list of author information is available at the end of the article
}

psychologic morbidity $[15,16]$, and reduced quality of life $[8,10,17]$. Early surgical nerve repair leads to better functional recovery in the upper limb $[8,9,17,18]$.

Clinical examination is not accurate in brachial plexus injuries owing to the complicated plexus design and also the complex nature of the lesions. EMG provides information about the functional involvement, not the localization of the lesion [19]. The delay from injury to surgical repair is the leading cause of poor outcome [20-22].

Pre-surgical diagnosis is now important because of the improvements in the surgical field. As MRI is a noninvasive, non-radiative imaging modality with the multiplanar capability and better soft tissue characterization, it becomes a modest diagnostic imaging modality [19]. The current study aims to evaluate the role of MRI in 
the diagnosis of adult traumatic and obstetric brachial plexus injury comparing its results with intraoperative findings.

\section{Methods}

\section{Study participants}

A prospective cross-sectional study was done from December 2016 to June 2019. The study protocol was approved by the Ethics Committee, and informed consent was obtained from every patient or their parents (in the obstetric group).

A total of 46 patients with brachial plexus injury (traumatic and obstetric brachial plexus injury) were recruited in this study. Intraoperative findings were available in 37 patients, so they were included. Clinical manifestations included neurological deficits of the shoulder and upper limb (motor, sensory, and reflexes).

\section{Inclusion criteria}

The examination was performed in patients presented with traumatic or obstetric brachial plexus injury regardless of the age of the case.

\section{Exclusion criteria}

In the current study, any patients who were contraindicated for MRI examination have been excluded as those who have an artificial cardiac pacemaker or metallic prosthesis are not compatible with MRI. Also, those with severe claustrophobia were excluded. Also, we excluded patients with a previous history of brachial plexus operative exploration.

\section{MR image acquisition}

MRI was performed with Siemens Sempra 1.5 T superconducting MRI scanner, with an 8-channel neurovascular coil. Patients were scanned in the supine position with the arms positioned beside the body in a neutral position and the head entering the scanner first. Patients were asked to reduce swallow activity and body movement during the scanning.

\section{MR sequences}

Our protocol includes the following: sagittal T1WITR/TE 400/7.8 ms, FOV $200 \mathrm{~mm}$, and acquisition time 4:30 min; sagittal STIR-TR/TE 3200/80 ms, FOV 200 $\mathrm{mm}$, and acquisition time 2:40 min; axial and coronal T2WI-TR/TE 3000/120 ms, FOV $200 \mathrm{~mm}$, and acquisition time 3:30 min; coronal 3D T2 STIR SPACETR/TE $3000 / 183 \mathrm{~ms}$, FOV $240 \mathrm{~mm}$, and acquisition time 4:00 min; coronal DWIBS-TR/TE 2700/63 ms, FOV $370 \mathrm{~mm}$, and acquisition time 0:54 min; and radial coronal myelography-TR/TE $8000 / 793 \mathrm{~ms}$, FOV $280 \mathrm{~mm}$, and acquisition time $1: 54 \mathrm{~min}$. The total acquisition time is $20: 58 \mathrm{~min}$.
The scanning range was from the upper edge of the 4th cervical vertebral body down to the lower edge of the 2nd thoracic vertebral body, and from the posterior edge of the spinal canal to the sternoclavicular joint. Both sides including the armpit were included. The slice thickness was $3 \mathrm{~mm}$. Sagittal views were taken parallel to the curvature of the cervical spine. Axial views were dead axial, while coronal views were dead coronal involving both sides for comparison especially in T2 STIR sequences. There is no need to take oblique views as brachial plexus had shallow obliquity and the entire segments of the brachial plexus were shown especially in the 3D T2 STIR SPACE sequence.

\section{Image processing and analysis}

The original images were obtained by conventional sequences (T1WI and T2WI), DWIBS sequence scanning, 3D T2 STIR SPACE, and MR radial myelography which were imported into Siemens MR Workspace for 3D reconstruction. The maximum intensity projection (MIP) was reconstructed. The images were reviewed and analyzed by 2 same senior radiologists (of 15 and 20 years of experience); then, MRI findings were compared with the intraoperative data which were revealed during intraoperative exploration.

The intraoperative evaluation was considered as a gold standard to compare the MRI findings with it. The study was conducted in a double-blind manner.

\section{Statistical analysis}

Statistical analysis was carried out with the SPSS software 23.0 version. Continuous data are expressed as mean $\pm \mathrm{SD}$, while categorical data were expressed as numbers and percentages. MRI findings were compared with intraoperative findings as a gold standard for the calculation of sensitivity, specificity, and accuracy. Data regarding the agreement between different diagnostic methods (different MRI sequences and operative findings) was based on the Cohen kappa test. Kappa values were weighted in intervals: < 0.2 poor, $0.21-0.4$ fair, 0.41-0.60 moderate, $0.61-0.80$ good, $0.81-0.99$ excellent, and 1 perfect. SPSS ver. 23 (SPSS Inc., Chicago, IL, USA) was used, and $p$ value of less than 0.005 was considered significant (Altman DG., 1995).

\section{Results}

MRI examination of brachial plexus was done for 37 patients with clinically suspected traumatic or obstetric brachial plexopathy. Those patients were surgically explored with confirmed intraoperative findings. Twentytwo patients were presented with traumatic brachial plexus injury: 20 males and 2 females, mean age 26.3 years ( $7-51$ years). The other 15 patients were presented with obstetric brachial plexus injury: 10 males and 5 
females, mean age 12.8 months (7-28 months). Nineteen cases were presented with a right-sided injury, while 18 cases were presented with a left-sided injury.

Intraoperative examination revealed that 26 patients had a preganglionic injury in 69 nerves, and 24 patients had postganglionic injury seen in 56 segments (classified as follows: 8 patients have isolated preganglionic injury, 6 patients have isolated postganglionic injury, 18 patients have both preganglionic and postganglionic injury together, and 5 patients were free with intact brachial plexus).

For preganglionic injury, in each patient, 5 nerve roots (from C5-T1) were examined; total preganglionic segments $5 \times 37=185$ segments. In postganglionic injury, in each patient, 17 segments were examined (5 roots, 3 trunks, 6 divisions, and 3 cords); total postganglionic segments $17 \times 37=629$ segments.

\section{MRI findings}

Preganglionic nerve injury (avulsion) was manifested either as empty neuroforamen or as pseudomeningocele formation or interrupted nerve continuity (Figs. 1 and 2). As regards the postganglionic injuries, they were manifested in different patterns: (a) nerve rupture, appearing as different degrees of nerve thickening with abnormal high signals especially in STIR sequence, or (b) neuroma formation, appearing as focal thickening of the injured segment of the nerve (Figs. 1, 2, and 3).

\section{Correlation with intraoperative findings}

For the preganglionic injury, 69 nerve roots were surgically explored and confirmed to be avulsed; MRI identified 60 of them, so the sensitivity of MRI in detecting preganglionic injury was $86 \%$, with specificity $92 \%$ and accuracy $90 \%$ with good agreement between MRI results

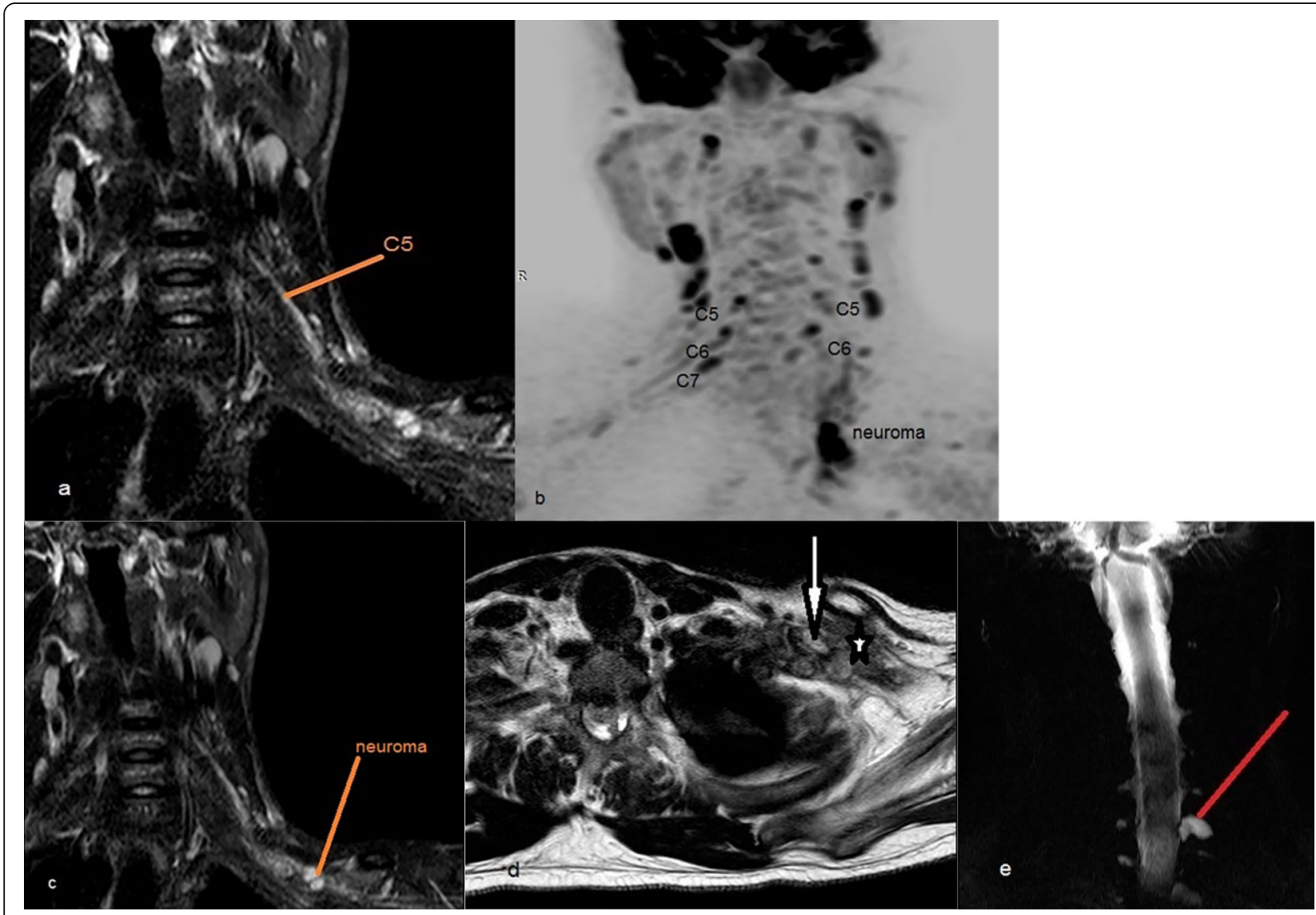

Fig. 1 A 24-year-old male patient presented with left traumatic brachial plexus injury following MBA 3 months before imaging. a MIP processing of coronal STIR shows high signal and thickened left C5 and focal thickening at the level of the left-sided cords. $\mathbf{b}$ MIP of coronal DWIBS shows thickened left C5 and C6 with restricted signal and traumatic neuroma. c Coronal STIR shows retracted avulsed nerves with a focal thickening at the level of the cords. $\mathbf{d}$ Axial T2WI shows focal thickening at the level of the cords (white arrow) and retracted nerves (*). e Radial myelography showing pseudomeningocele formation at T1 level. Intraoperative data are ruptured left-sided C5 and C6; avulsed C7, C8, and T1; and traumatic neuroma formation at the level of cords of the brachial plexus with adhesions around after operative repair of an injured subclavian artery immediately after the trauma 


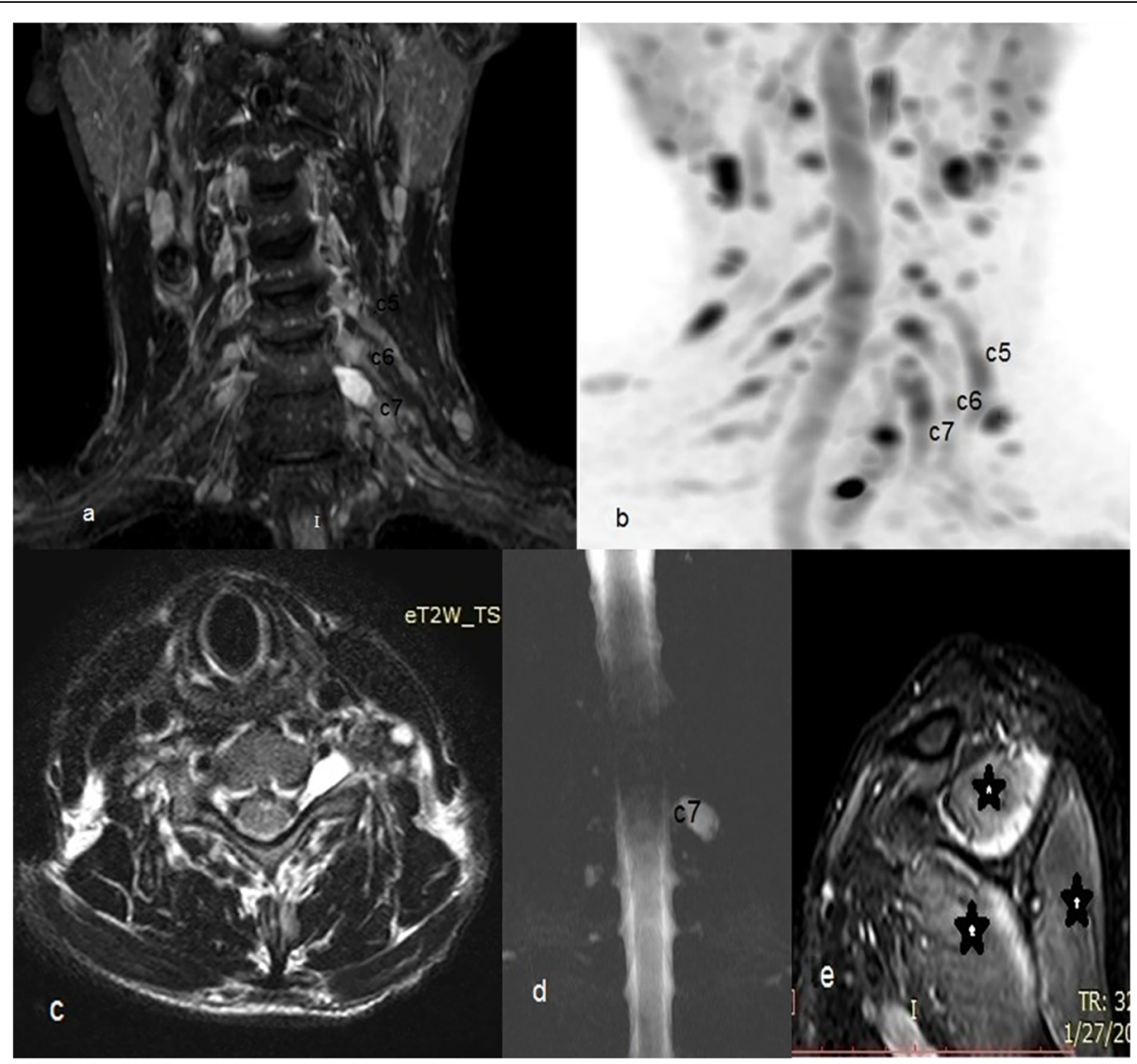

Fig. 2 A 55-year-old male patient presented with left side traumatic brachial plexus injury following MCA 6 months before imaging. a MIP processing of coronal STIR shows thickened left C5 and C6 with increased signal and pseudomeningocele at left C7. b MIP of coronal DWIBS shows thickened left C5, C6, and C7 with a restricted signal. c Axial T2WI and $\mathbf{d}$ radial myelography showing pseudomeningocele formation at left C7. e Sagittal STIR shows edema of the left rotator cuff muscles (stars). Intraoperative data are ruptured left C5 and C6 with avulsed C7

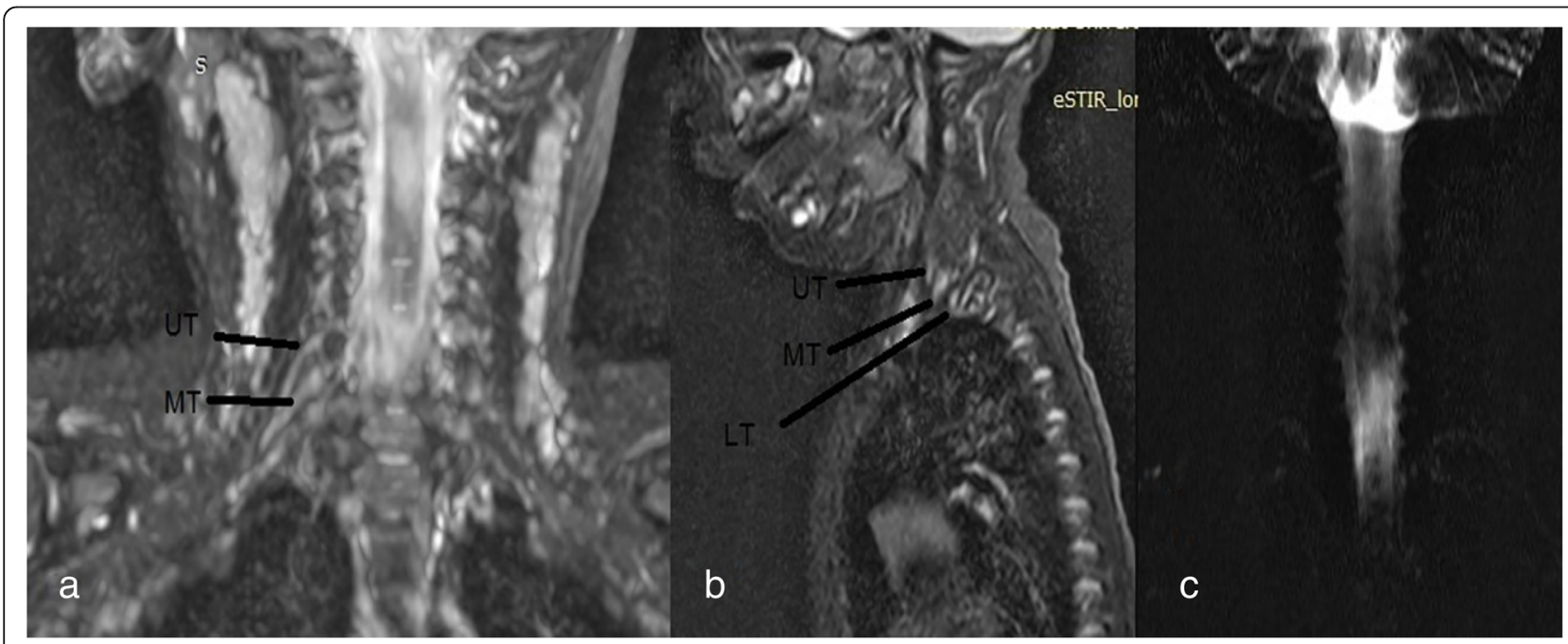

Fig. 3 An 11-month-old male patient presented with right-sided obstetric brachial plexus injury. a MIP of coronal STIR and b sagittal STIR showing mildly thickened with an increased signal of the upper trunk (UT) and middle trunk (MT) compared to the lower trunk (LT) of the right brachial plexus. c Radial myelography showing no meningocele formation at right-sided neuroforamina. Operative data are partially injured rightsided upper and middle trunks 
and operative findings $\kappa=0.792, p<0.005$. Nerve root avulsion was detected in MRI by (a) pseudomeningocele formation which was observed in 39 roots $(65 \%)$, or (b) interrupted nerve continuity which was observed in 11 nerve roots (18\%), and (c) empty neuroforamen which was detected in 10 nerve roots (16.6\%) (Table 1).

For postganglionic injury, there were injured 56 segments (41 ruptured segments with 15 segments having neuroma formation) as confirmed intraoperatively; MRI could detect 30 of the total 41 ruptured segments, and 11 segments with neuroma formation of the total 15 segments. Thus, in nerve rupture, MRI had $73 \%$ sensitivity, 99\% specificity, and 90\% accuracy with excellent agreement between MRI results and operative findings $K=$ $0.811, p<0.005$, while in neuroma formation, MRI had $73 \%$ sensitivity, $100 \%$ specificity, and $99 \%$ accuracy with excellent agreement between MRI results and operative findings $\kappa=0.843, p<0.005$ (Table 1 ).

\section{Subgroup analysis}

MRI results showed a difference between obstetric and adult brachial plexus injury, where MRI had higher sensitivity and accuracy in adult patients.

\section{Obstetric brachial plexus injury}

Preganglionic injury There were avulsed 19 nerve roots proven by intraoperative findings, and only 12 of them were detected by MRI, thus lower MRI sensitivity, specificity, and accuracy in preganglionic injury.

Postganglionic injury The ruptured nerve segments were intraoperatively detected in 23 segments where MRI detected only 14 of them, so MRI results for obstetric nerve rupture were $60 \%$ sensitivity, $99 \%$ specificity, and $95 \%$ accuracy. In the case of traumatic neuroma formation, there were 10 segments which showed neuroma formation where MRI detected 8 of them with sensitivity $80 \%$, specificity $100 \%$, and accuracy $99 \%$ (Table 2 ).

\section{Traumatic brachial plexus injury}

Preganglionic injury Intraoperative data revealed 50 avulsed nerve roots, and MRI detected 48 of them with $96 \%$ sensitivity, $95 \%$ specificity, and $95 \%$ accuracy.

Postganglionic injury There were ruptured 18 nerves and MRI detected 16 of them, so its results were $88 \%$ sensitivity, 100\% specificity, and 99\% accuracy, while post-traumatic neuroma affected 5 segments and MRI detected only 3 of them with sensitivity, specificity, and accuracy of $60 \%, 100 \%$, and $99 \%$, respectively (Table 2 ).

\section{Discussion}

Magnetic resonance neurography is increasingly being accepted as the investigation of choice in lesions affecting the brachial plexus and the peripheral nerves [23].

We found that MRI had high diagnostic performance in brachial plexus injury and the subgroup analysis with better sensitivity and accuracy in adult cases.

In this study, MRI sensitivity and specificity in root avulsion in obstetric brachial plexus injury were $63 \%$ and $89 \%$, respectively. This is in agreement with a previous study conducted by Somashekar et al. [24], who included 13 patients. They found that the sensitivity and specificity of MRI for nerve root avulsions were $75 \%$ and $82 \%$, respectively.

The current study revealed that MRI sensitivity and specificity in the detection of traumatic neuroma formation in obstetric cases were $80 \%$ and $100 \%$, respectively. A previous study by Medina et al. [25] stated that sensitivity and specificity of MRI in post-traumatic neuroma were $97 \%$ and $100 \%$, respectively, and their study was

Table $1 \mathrm{MRI}$ diagnostic results in detection of preganglionic and postganglionic injury in all cases including adult and obstetric cases compared to operative findings

\begin{tabular}{|c|c|c|c|c|c|c|c|}
\hline \multirow[t]{2}{*}{ MRI } & \multicolumn{2}{|c|}{ Operative } & \multirow{2}{*}{$\begin{array}{l}\text { Sensitivity } \\
\text { (\%) }\end{array}$} & \multirow{2}{*}{$\begin{array}{l}\text { Specificity } \\
\text { (\%) }\end{array}$} & \multirow{2}{*}{$\begin{array}{l}\text { Accuracy } \\
\text { (\%) }\end{array}$} & \multirow[t]{2}{*}{ Kappa } & \multirow{2}{*}{$\begin{array}{l}p \\
\text { value }\end{array}$} \\
\hline & Yes & No & & & & & \\
\hline \multicolumn{8}{|c|}{ Avulsion } \\
\hline Yes & 60 & 9 & 86 & 92 & 90 & 0.792 & 0.000 \\
\hline No & 9 & 107 & & & & & \\
\hline \multicolumn{8}{|c|}{ Rupture } \\
\hline Yes & 30 & 2 & 73 & 99 & 97 & 0.811 & 0.000 \\
\hline No & 11 & 586 & & & & & \\
\hline \multicolumn{8}{|c|}{ Neuroma } \\
\hline Yes & 11 & 0 & 73 & 100 & 99 & 0.843 & 0.000 \\
\hline No & 4 & 614 & & & & & \\
\hline
\end{tabular}


Table 2 Comparison of MRI diagnostic performance in obstetric and adult traumatic brachial plexus injury as compared to operative findings

\begin{tabular}{lll}
\hline & Obstetric cases & Adult cases \\
\hline Avulsion & & \\
Number & $12 / 19$ & $48 / 50$ \\
Sensitivity & $63 \%$ & $96 \%$ \\
Specificity & $89 \%$ & $95 \%$ \\
PPV & $66 \%$ & $94 \%$ \\
NPV & $87 \%$ & $96 \%$ \\
Edema & & \\
Number & $14 / 23$ & $16 / 18$ \\
Sensitivity & $60 \%$ & $88 \%$ \\
Specificity & $99 \%$ & $100 \%$ \\
PPV & $87 \%$ & $100 \%$ \\
NPV & $96 \%$ & $99 \%$ \\
Neuroma & & \\
Number & $8 / 10$ & $3 / 5$ \\
Sensitivity & $80 \%$ & $60 \%$ \\
Specificity & $100 \%$ & $100 \%$ \\
PPV & $100 \%$ & $100 \%$ \\
NPV & $99 \%$ & $99 \%$ \\
\hline
\end{tabular}

$M R I$ magnetic resonance imaging, $P P V$ positive predictive value, $N P V$ negative predictive value

done with a larger sample size (31 children) than our study.

In our study, MRI results in traumatic brachial plexus injury in adults were 96\% sensitivity, 95\% specificity, and 95\% accuracy for preganglionic lesions, in agreement with a study conducted by Zhang et al. [26], who concluded that MRI sensitivity, specificity, and accuracy were $93 \%, 71 \%$, and $89 \%$, respectively, for the preganglionic injury. The same study revealed that in postganglionic injury, MRI had $91 \%$ sensitivity, $60 \%$ specificity, and $85 \%$ accuracy, which were higher than our results with $88 \%$ sensitivity, $100 \%$ specificity, and $99 \%$ accuracy for nerve rupture and $60 \%$ sensitivity, $100 \%$ specificity, and 99\% accuracy for neuroma formation. This may be owing to the usage of MRI $3 \mathrm{~T}$ in the former study while we used MRI $1.5 \mathrm{~T}$ in our study.

In our study, it is clear that MRI results in adult cases were better than those in obstetric cases and this is suggested to be due to several factors including the larger field of view and more fat planes in neck spaces in adult cases so larger spaces in between the examined nerves with better control of breathing movements in adult than infants so better image resolution.

In agreement with Vargas et al. [27], the current study data showed that radiological examination by MRI $1.5 \mathrm{~T}$ using the previously described sequences, the conventional sequences (T1WI, T2WI, and STIR) and the advanced sequences (3D STIR SPACE, DWIBS, and MR myelography), and its interpretation by an expert radiologist provides the best diagnostic accuracy for brachial plexus injury (either adult traumatic or obstetric injury) when its results were compared to intraoperative findings.

Adding DWIBS sequence does not extend the examination excessively, only by approximately $5 \mathrm{~min}$. When combined with the other sequences and planes of conventional MRI of the brachial plexus, this examination can provide a more accurate assessment without increasing morbidity [28].

Diffusion-weighted imaging with background subtraction (DWIBS) provides greater contrast between the nerves and adjacent tissues, improving the image of the nerve pathways in the brachial plexus along its entire course not only the avulsed root [28].

In this study, performing 3D T2 STIR SPACE sequence allows clear visualization of the neuroforamina either empty or not (compared to the other contralateral intact brachial plexus) especially if no pseudomeningocele was formed so improving the diagnosis of root avulsion.

From the overall results, it is clear that MRI provides a safe, accurate non-invasive imaging modality for preoperative assessment of traumatic brachial plexus injury in adult and obstetric cases as well.

Up to our knowledge, there are no previous available studies comparing the adult and obstetric BPI and our study is the first study to investigate that.

Our study is not just a descriptive study, but an analytic one, calculating the sensitivity, specificity, accuracy, and agreement with operative data.

Given the relatively small size and subgroup heterogeneity, we recommend reproducing such work with a larger sample and focusing on either adult or obstetric cases.

\section{Conclusion}

MRI is the imaging modality of choice in the examination of traumatic and obstetric brachial plexus injuries; it is safe and non-invasive, having the multiplanar capability and better soft tissue characterization.

\section{Abbreviations}

DWIBS: Diffusion-weighted imaging with background subtraction;

EMG: Electromyogram; FOV: Field of view; LT: Lower trunk; MBA: Motorbike accident; MCA: Motor car accident; MRI: Magnetic resonance imaging; MT: Middle trunk; NPV: Negative predictive value; PPV: Positive predictive value; SPACE: Sampling Perfection with Application optimized Contrasts using different flip angle Evolution; STIR: Short tau inversion recovery; UT: Upper trunk

\section{Acknowledgements}

The authors thank all the study participants for their patience and support. 


\section{Ethical considerations}

1. Risk benefit assessment for all patients was indicated for the examination during this study.

2. Confidentiality: all patients' data were confidentially kept.

3. The research was done by scientifically qualified and trained personnel only.

4. The procedures included in this study had been already used in hospital and centers in and outside Egypt.

\section{Authors' contributions}

D.M.G., M.T.H, and M.M.K. suggested and developed the research idea, and reviewed the literature. D.M.G. and M.A. were responsible for the data collection and analysis, performed the statistical analysis, wrote and revised the manuscript, prepared the cases, performed the required measurements, and prepared the figures and tables. D.M.G. and H.A.Y. were responsible for reporting the cases of MRI and referred to us a result to compare it with operative findings. The authors have a major contribution in preparing and editing the manuscript. The authors read and approved the final manuscript.

\section{Funding}

This study was financially supported by the Grant Office of the Faculty of Medicine, Assiut University.

\section{Availability of data and materials}

The data that support the findings of this study are available from Radiology Department, Assiut University, but there are restrictions that apply to the availability of data, which were used under license for this study, and so were not publicly available. Data were available from authors upon request with permission of the head of the Radiology Department, Assiut University.

\section{Ethics approval and consent to participate}

This study had approval from the Faculty of Medicine Research Ethics Committee, Assiut University, Egypt. All patients who participated in this study signed an informed written consent for participation.

\section{Consent for publication}

All patients included in this research gave written informed consent to publish the data contained within this study

\section{Competing interests}

Authors declare that they had no competing interests.

\section{Author details}

${ }^{1}$ Department of Radiology, Assiut University, Assiut, Egypt. ${ }^{2}$ Department of Orthopedic Surgery and Traumatology, Assiut University, Assiut, Egypt.

Received: 16 July 2020 Accepted: 8 September 2020 Published online: 24 September 2020

\section{References}

1. Grahn P, Pöyhiä T, Sommarhem A, Nietosvaara Y (2019) Clinical significance of cervical MRI in brachial plexus birth injury. Acta Orthop 90(2):111-118

2. Foad SL, Mehlman CT, Ying J (2008) The epidemiology of neonatal brachial plexus palsy in the United States. J Bone Joint Surg Am 90(6):1258-1264

3. Pöyhiä $T H$, Lamminen $A E$, Nietosvaara $Y(2010)$ Brachial plexus birth injury: US screening for glenohumeral joint instability. Radiology 254(1):253-260

4. Eisman EA, Little KJ, Laor T, Cornwall R (2015) Glenohumeral abduction contracture in children with unresolved neonatal brachial plexus palsy. J Bone Joint Surg Am 97(2):112-118

5. Gharbaoui IS, Gogola GR, Aaron DH, Kozin SH (2015) Perspectives on glenohumeral joint contractures and shoulder dysfunction in children with perinatal brachial plexus palsy. J Hand Ther 28(2):176-184

6. Little KJ, Zlotolow DA, Soldado F (2014) Early functional recovery of elbow flexion and supination following median and/or ulnar nerve fascicle transfer in upper neonatal brachial plexus palsy. J Bone Joint Surg Am Vol 96:215221. https://doi.org/10.2106/JBJS.L.01405

7. Wade RG, Takwoingi Y, Wormald JC (2019) MRI for detecting root avulsions in traumatic adult brachial plexus injuries: a systematic review and metaanalysis of diagnostic accuracy. Radiology 293(1):125-133
8. Franzblau LE, Shauver MJ, Chung KC (2014) Patient satisfaction and selfreported outcomes after complete brachial plexus avulsion injury. J Hand Surg Am 39(5):948-55.e4

9. Kretschmer T, Ihle S, Antoniadis $\mathrm{G}$ et al (2009) Patient satisfaction and disability after brachial plexus surgery. Neurosurgery 65(4 Suppl):A189-A196

10. Dolan RT, Butler JS, Murphy SM, Hynes D, Cronin KJ (2012) Health-related quality of life and functional outcomes following nerve transfers for traumatic upper brachial plexus injuries. J Hand Surg Eur 37(7):642-651

11. Maldonado AA, Kircher MF, Spinner RJ, Bishop AT, Shin AY (2016) Free functioning gracilis muscle transfer versus intercostal nerve transfer to musculocutaneous nerve for restoration of elbow flexion after traumatic adult brachial pan-plexus injury. Plast Reconstr Surg 138(3):483e-488e

12. Teixeira MJ, da Paz MGDS, Bina MT, Santos SN, Raicher I, Galhardoni R, De Andrade DC. Neuropathic pain after brachial plexus avulsion-central and peripheral mechanisms. BMC neurology. 2015;15(1):1-9.

13. Lee K (2012) Long term effect of thalamic deep brain stimulation for pain due to brachial plexus injury. J Pain 13(4 Suppl):S68

14. Brill S, Aryeh IG (2008) Neuromodulation in the management of pain from brachial plexus injury. Pain Physician 11(1):81-85

15. Franzblau L, Chung KC (2015) Psychosocial outcomes and coping after complete avulsion traumatic brachial plexus injury. Disabil Rehabil 37(2): 135-143

16. Wilson TJ, Chang KWC, Yang LJS (2016) Depression and anxiety in traumatic brachial plexus injury patients are associated with reduced motor outcome after surgical intervention for restoration of elbow flexion. Neurosurgery 78(6):844-850

17. Mancuso CA, Lee SK, Dy CJ, Landers ZA, Model Z, Wolfe SW (2015) Expectations and limitations due to brachial plexus injury: a qualitative study. Hand (N Y) 10(4):741-749

18. Liu Y, Lao J, Gao K, Gu Y, Zhao X (2013) Functional outcome of nerve transfers for traumatic global brachial plexus avulsion. Injury 44(5):655-660

19. Balakrishna S (2019) January) A pictorial essay of MRI findings-obstetric brachial plexopathy. Eur Congress Radiol 2019

20. Jivan S, Novikova LN, Wiberg M, Novikov LN (2006) The effects of delayed nerve repair on neuronal survival and axonal regeneration after seventh cervical spinal nerve axotomy in adult rats. Exp Brain Res 170(2):245-254

21. Jivan S, Kumar N, Wiberg M, Kay S (2009) The influence of pre-surgical delay on functional outcome after reconstruction of brachial plexus injuries. J Plast Reconstr Aesthet Surg 62(4):472-479

22. Martin E, Senders J, DiRisio A, Smith T, Broekman M (2019) Timing of surgery in traumatic brachial plexus injury: a systematic review. J Neurosurg 130(4):1333-1345

23. Upadhyaya V, Upadhyaya D, Mishra B (2018) MR neurography in traumatic, non-obstetric paediatric brachial plexopathy. Eur Radiol 28(6):2417-2424

24. Somashekar D, Yang L, Ibrahim M, Parmar H (2013) High-resolution MRI evaluation of neonatal brachial plexus palsy: a promising alternative to traditional CT myelography. Am J Neuroradiol 35(6):1209-1213

25. Medina L, Yaylali I, Zurakowski D, Ruiz J, Altman N, Grossman J (2006) Diagnostic performance of MRI and MR myelography in infants with a brachial plexus birth injury. Pediatr Radiol 36(12):1295-1299

26. Zhang, L., Xiao, T., Yu, Q., et al. (2018). Clinical value and diagnostic accuracy of 3.0 T multi-parameter magnetic resonance imaging in traumatic brachial plexus injury. Medical science monitor: international medical journal of experimental and clinical research, 24, 7199.

27. Vargas MI, Gariani J, Delattre BA et al (2015) 3D MR imaging of the brachial plexus. Semin Musculoskelet Radiol 19:137-148

28. Veronesi BA, Rodrigues MB, Sambuy MT et al (2018) Use of magnetic resonance imaging to diagnose brachial plexus injuries. Acta Ortopedica Brasileira. Mar-Apr 26(2):131-134

\section{Publisher's Note}

Springer Nature remains neutral with regard to jurisdictional claims in published maps and institutional affiliations. 